\title{
Fractura periprotésica acetabular intraoperatoria
}

\author{
Intraoperative acetabular periprotesic fracture
}

\author{
Marqués-López F,* Nieto-Alvarado S, ${ }^{\ddagger}$ Tey-Pons M, ${ }^{\S}$ León-García-Vao A, ${ }^{\S}$ Molina-Cuevas LC \\ Unidad de Cadera Hospital del Mar.
}

RESUMEN. Las fracturas intraoperatorias acetabulares (FIA) son una complicación rara con incidencia de 2 a 5\%. Presentamos la revisión de una serie de 13 casos, que aborda diagnóstico, tratamiento y resultados. Material y métodos: entre 2006 y 2018 realizamos 4,800 artroplastías primarias de cadera (ATC). En nuestro hospital identificamos 13 casos con FIA con un seguimiento de 38.3 meses. Resultados: Dos casos fueron estables con manejo conservador; 11 casos inestables: en siete casos colocamos trabecular metal, en un caso aumentamos diámetro acetabular, en un caso recambio de cotilo, en un caso revisión con anillo tipo Ganz y un caso no aceptó tratamiento. El uso de trabecular metal es un implante que ayuda eficazmente y con buenos resultados en FIA. Conclusión: Estas fracturas son raras, conocer el implante a utilizar es imperativo, ya que si los implantes están sobredimensionados, con mayor rigidez o hemisféricos, los pacientes tienen mayor riesgo de sufrir esta complicación.

Palabras clave: Fractura, acetabular, prótesis de cadera, tratamiento.

\section{Introducción}

La artroplastía total de cadera (THA) tiene un éxito a 10 años en $90 \%$ de los pacientes, ${ }^{1}$ en Estados Unidos se espera que la THA aumente $175 \%$ para 2030, en comparación
ABSTRACT. Intraoperative acetabular fractures (IAF) is a rare complication with an incidence $2 \%-5 \%$. We present the review of a series of 13 cases, discussing diagnosis, treatment and results. Material and methods: Between 2006 and 2018, we performed 4800 primary hip arthroplasties (THA) In our hospital, we identified 13 cases with FIA with a follow-up of 38.3 months. Results: Two cases were stable with conservative management; 11 unstable cases: seven cases we placed trabecular metal, one case we increased acetabular diameter, one case acetabular replacement, one case revision with Ganz type ring and one case do not accept treatment. The use of trabecular metal is an implant that helps effectively and with good results in FIA. Conclusion: These fractures are rare, knowing the implant to use is mandatory because if the implants are oversized, with greater rigidity or hemispheric, patients have increased risk of suffering from this complication.

Keywords: Fracture, acetabular, hip prosthesis, treatment.

con $2005,{ }^{2}$ con un aumento en la tasa de revisiones de hasta 137\% para 2030 por múltiples fallas. ${ }^{2,3}$

Springer y colaboradores encontraron que la inestabilidad es la causa principal de revisión de THA (35\%), seguida de aflojamiento aséptico (30\%) e infección (12\%). ${ }^{4}$

\section{Nivel de evidencia: IV}

\footnotetext{
* Jefe del Departamento de Cirugía Reconstructiva y Trauma de Cadera. Unida de Cadera Hospital del Mar.

* Médico residente de quinto año de Cirugía Articular.

§ Adscrito de Cirugía Reconstructiva y Trauma de Cadera. Unidad de Cadera Hospital del Mar.

^ Médico Interno de pregrado Unidad Hospitalaria IMSS Clínica No. 2.

Correspondencia:

Dr. Fernando Marqués-López Md.Phd.

Passeig Maritim 25-29, Barcelona, España, C.P. 08003.

E-mail: fmarqueslopez@gmail.com
}

Recibido: 10-07-2020. Aceptado: 25-08-2021. 
Las fracturas intraoperatorias del acetábulo son una complicación extremadamente rara, puede producirse durante la exposición acetabular, luxación cadera transquirúrgica, fresado del acetábulo o impactación del cotilo press fit. Estudios previos demuestran que el fresado excesivo del acetábulo y la impactación de un componente acetabular sobredimensionado puede predisponer a una fractura intraoperatoria. ${ }^{5,6,7,8}$

La incidencia de fracturas periprotésicas va en aumento debido a mayor prevalencia de artroplastía total de cadera y al uso de técnicas de fijación sin cemento. ${ }^{9}$ De todas las revisiones de THA encontramos que $2-5 \%$ se deben a fracturas periprotésicas acetabulares. ${ }^{1,10}$ Con una prevalencia de $<0.02 \%$ en prótesis cementadas y de $0.4 \%$ en prótesis no cementadas, la elección de implante demostró que el uso de un cotilo monoblock aumenta hasta 3.5\% el riesgo y con el uso de hemisféricos disminuye a $0.009 \%{ }^{11}$

Factores de riesgo: pacientes que padezcan comorbilidades como artritis reumatoide, ${ }^{6}$ osteoporosis, osteopenia, ${ }^{6,12}$, antecedentes de infiltraciones acetabulares, ${ }^{12}$ enfermedad de Paget. ${ }^{13}$

Fracturas intraoperatorias: Callaghan y colaboradores en un estudio in vitro reportaron una tasa de fractura superior a 50\% cuando el sobrefresado era de más de $4 \mathrm{~mm} \mathrm{y}$ de $12 \%$ cuando se limitó a $2 \mathrm{~mm} \cdot{ }^{14}$ Las fracturas también pueden ocurrir debido a una combinación de exceso fresado y fuerza de impacto de la copa en presencia de un acetábulo debilitado o deficiente debido a la osteólisis u osteoporosis. Más comúnmente situada en el fondo del acetábulo.

Kim y colaboradores ${ }^{15}$ reportaron que en cadáveres con la inserción de copas sobredimensionadas $>2$ y $4 \mathrm{~mm}$ aumentaban las fracturas acetabulares, así como el uso de una fuerza de impactación de 500 a 1,000 N no logra bajar un cotilo acetabular sobredimensionado, por lo que se aumenta la fuerza de 2,000 a 3,000 N para asentar el cotilo sobredimensionado de $>2$ a $4 \mathrm{~mm}$.

Las fracturas deben reconocerse durante la cirugía y evaluadas con una exposición amplia del sitio de fractura, reconocer las paredes y columnas afectadas. Debe ponerse énfasis en la importancia de la toma de radiografías intraoperatorias a pesar de la visualización directa de la fractura para comprobar su extensión. Las fracturas acetabulares son lesiones raras y se reporta que su manejo es complejo, ya que requiere comúnmente recambio de la copa acetabular. ${ }^{16}$

Clasificación: el sistema de clasificación de Vancouver de fracturas periprotésicas se puede utilizar en la evaluación y el manejo de fracturas periacetabulares en prótesis de cadera: ${ }^{17}$ grado I fractura no desplazada que no compromete el cotilo, grado II fractura no desplazada que compromete el cotilo potencialmente (fractura transversal del acetábulo o una fractura oblicua que separa la columna anterior y cúpula de la columna posterior), grado III fractura desplazada. Callaghan ${ }^{14}$ en un estudio in vitro incluyó fracturas de pared anterior, transversas, labio inferior y pared posterior. Pascarella ${ }^{18}$ clasifica de acuerdo con el momento traumático (Tabla 1).
Tratamiento: fractura intraoperatoria. El tratamiento conservador para implante estable consiste en ocho semanas sin descarga o realizar fijación con tornillos para aumentar estabilidad primaria del componente acetabular en diferentes orientaciones (íleon, isquion y pubis).

En implantes inestables con un desplazamiento de $2 \mathrm{~mm}$, el tratamiento es a base de reducción de fractura y fijación interna. El objetivo de la fijación será la estabilidad del implante. En algunos casos se colocan anillos de reforzamiento o copas cementadas y en algunos casos se requiere la revisión de implantes.

Fractura traumática. En implantes estables se da manejo conservador, ocho semanas sin descarga. Se requiere fijación interna si encontramos un fragmento impactado en el implante o inestabilidad del mismo.

En implantes inestables, se requiere considerar tratamiento con fijación interna con tornillos o placas, uso de anillo de reforzamiento, revisión de implante, megacopas, anillos de reforzamiento + injerto óseo.

Objetivo: el objetivo fue una revisión de la base de datos de nuestro hospital durante un período de 12 años, en el cual se revisaron todas las fracturas periprotésicas de acetábulo. Identificar factores de riesgo del paciente, la técnica quirúrgica realizada, el material protésico, su tratamiento y resultados funcionales.

\section{Material y métodos}

Entre 2006 y 2018 cinco cirujanos de cadera realizaron 4,800 artroplastías primarias de cadera, de las cuales se identificaron 13 casos que habían sufrido fractura intraoperatoria acetabular transquirúrgica $(0.27 \%)$. Los pacientes fueron localizados mediante nuestro registro total en fracturas periacetabulares; se estudiaron los motivos de la cirugía, de los cuales los más frecuentes fueron por osteoartritis, fracturas, secuelas de Perthes, Paget y epifisiólisis (Tabla 2).

Las copas acetabulares utilizadas fueron CSF plus en su mayoría, Trilogy, U Motion, FH Ortopedic, GT doble movilidad.

El diagnóstico de la fractura fue transquirúrgico y postquirúrgico con radiografía inmediata, en algunos no se logró estadificar la fractura, por lo cual se solicitó tomografía computarizada, su clasificación para tratamiento fue acorde

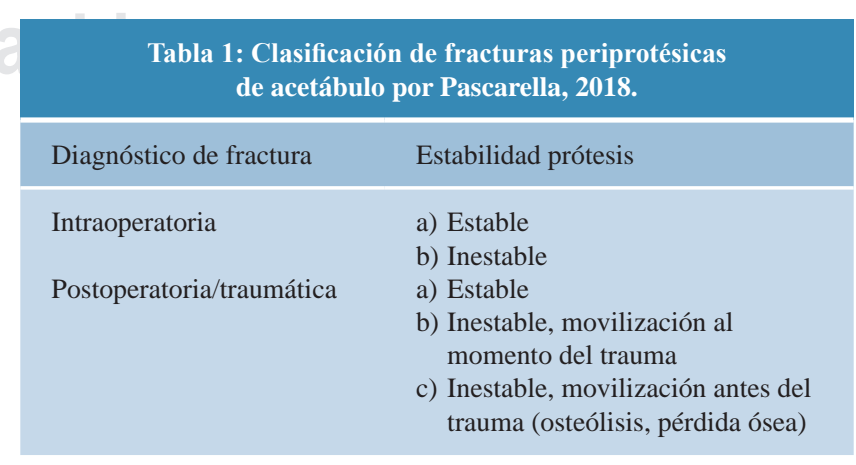




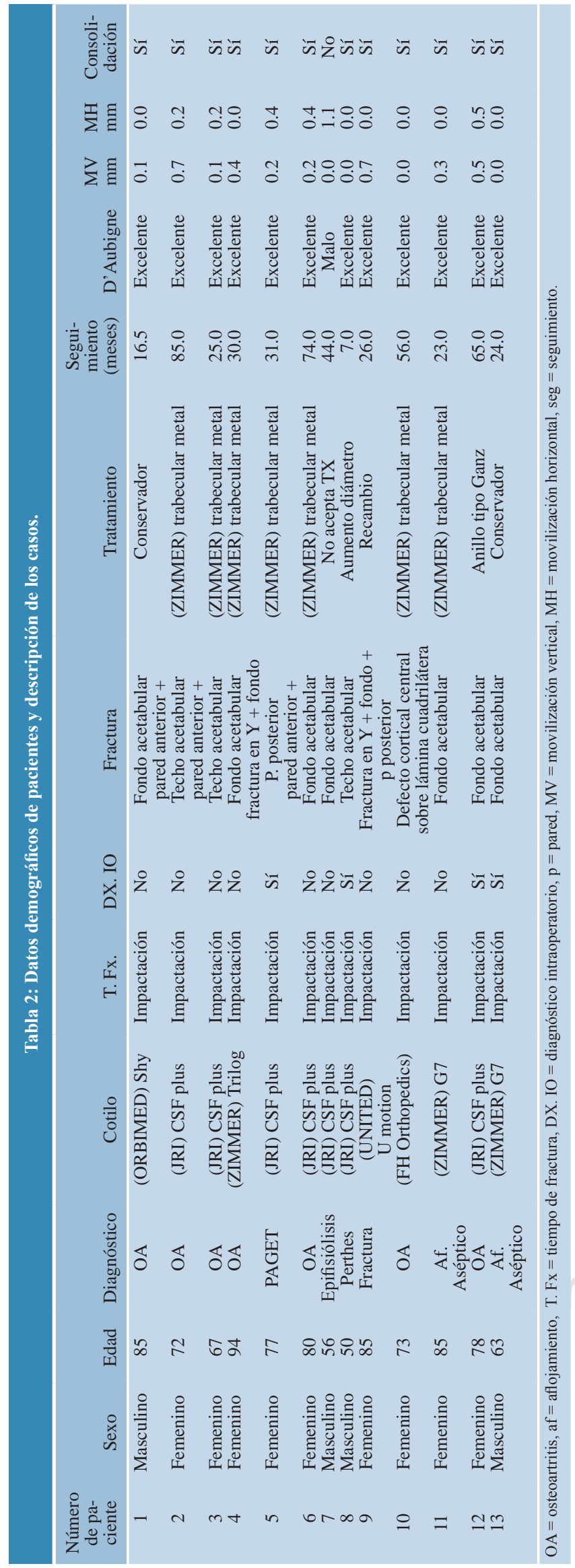

a Pascarella en estable e inestable utilizando como tratamiento quirúrgico para las estables reposo por ocho semanas y en las fracturas inestables el uso de placas, recambio de cotilo, aumento de diámetro de cotilo, copas jumbo y uso de anillos de reforzamiento.

La función fue registrada en las secciones de dolor, movilidad y marcha con la escala de Merle Postel D’Aubigne (MPD).

Se dio seguimiento radiológico dos, cuatro y ocho semanas y posteriormente de forma bimensual y anual evaluando la fijación de la copa, migración, integración y el grado de aflojamiento en caso de presentarse.

La migración acetabular radiológica fue con medidas trazando una línea horizontal que une la lágrima de la cadera al centro de la cabeza de la prótesis y el aflojamiento acetabular se definió como la migración del implante y presencia de zonas de radiolucencia e interfaz entre el implante-hueso o tornillo de fijación. Evaluamos la consolidación ósea según los criterios de Montoya así como la formación de hueso heterotópico (Tabla 2).

\section{Resultados}

El grupo incluyó nueve pacientes femeninos y cuatro masculinos, con una edad promedio de 75 años (rango de 50-94 años) en el momento de la cirugía, la distribución por lado afectado fue de siete derechas y seis izquierdas. Los diagnósticos preoperatorios fueron: siete osteoartritis de caderas, dos aflojamientos asépticos, uno por fractura subcapital, uno por secuelas de Perthes, uno por enfermedad de Paget y uno por secuelas de epifisiólisis, se realizaron 11 artroplastías primarias sin cementar y dos prótesis cementadas.

La distribución de los diseños de copa acetabular en los cuales se presentaron las fracturas fue la siguiente: monobloque esférico, siete CSF PLUS [7 JRI], un SHY [1 ORBIMED], un TRILOGY [1 ZIMMER] un U MOTION [1 UNITED], un FH ORTOPEDIC, dos G7 doble movilidad [ZIMMER], todos ellos hemisféricos de titanio.

En 12 de las 13 cirugías se realizó un abordaje lateral (hardinge) y sólo en uno abordaje posterorolateral), en dos de las caderas el fresado fue pressfit con fresado $<2 \mathrm{~mm}$ al componente definitivo utilizado y los otros 11 fueron line-to line. En todos los abordajes se utilizaron dos steinman 5.0, los cuales se colocan en el ilíaco en la parte superior a 2 cm del techo acetabular para mejor visualización, el diámetro de los cotilos acetabulares tuvo un promedio de $45 \mathrm{~mm}$ (rango 48-64 mm).

En cinco pacientes el diagnóstico fue transquirúrgico y en ocho fue postquirúrgico, a todos los pacientes se les tomó radiografía de control y en seis casos tomografía computarizada para estadificar la fractura.

La fractura más común fue el fondo acetabular en siete pacientes, tres con pared anterior + techo acetabular, dos casos con fractura en $\mathrm{Y}+$ fondo + p. posterior y un caso con defecto cortical central sobre lámina cuadrilátera. Clasificamos 

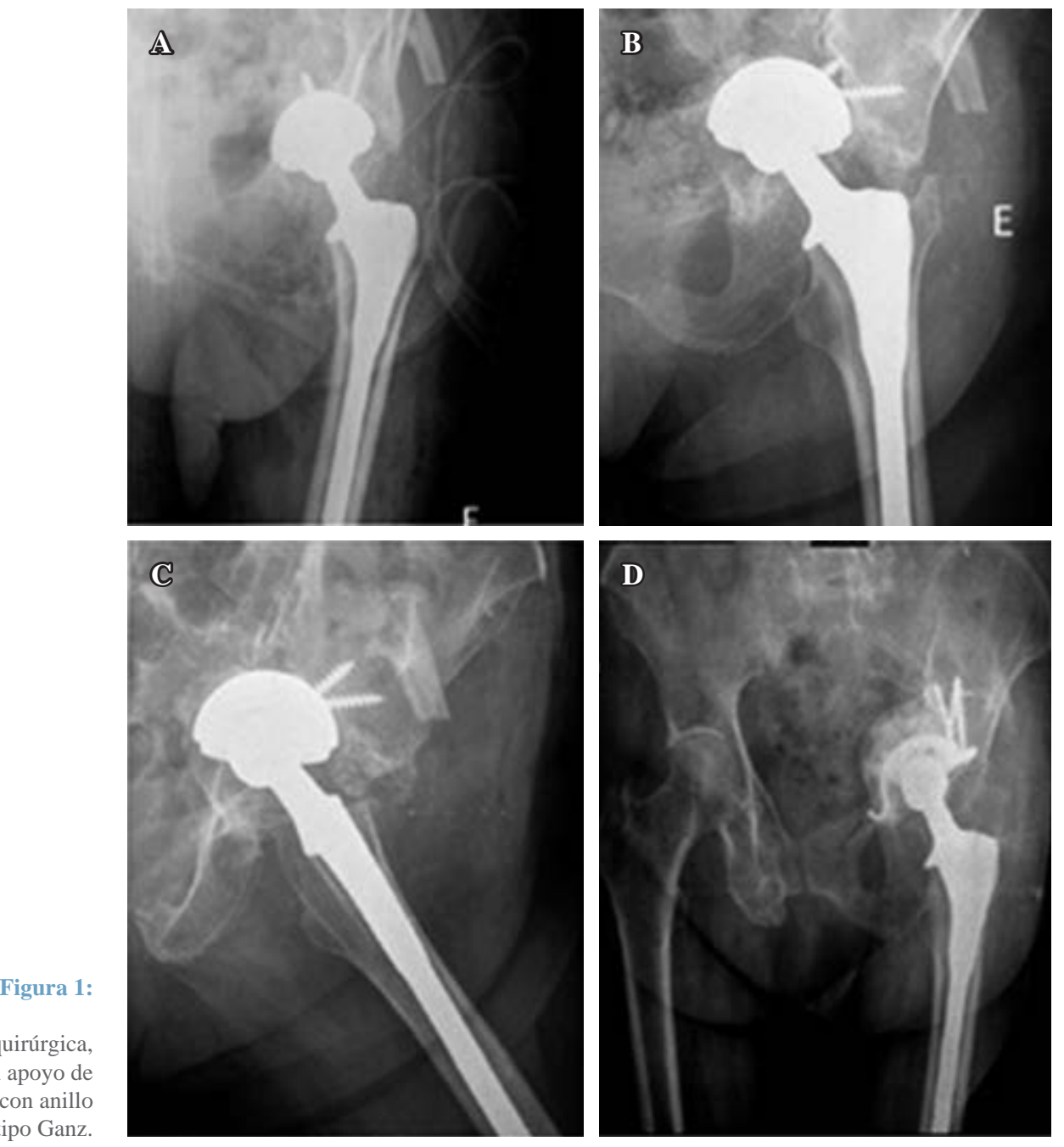

A) Protrusión acetabular transquirúrgica, B y C) resultado asociado al apoyo de la extremidad, D) resolución con anillo de refuerzo con gancho tipo Ganz.

las fracturas periprotésicas según Pascarella y se realizó el siguiente tratamiento: dos con cotilo estable (Cotilo Shy Orbimet y G7 Zimmer), en las cuales el manejo fue conservador por ocho semanas en reposo y 11 inestables, uno de los pacientes no aceptó tratamiento y en siete el manejo fue con sistema Trabecular Metal Acetabular Revision System (Figura 1), en uno se cambió cotilo CSF PLUS de 52 a 60 $\mathrm{mm}$, en uno se realiza recambio de cotilo U Motion por un Zimmer Doble Movilidad, se realiza revisión a un paciente con anillo tipo Ganz.

El seguimiento promedio fue de 38.3 meses (rango de uno a siete años), La escala de Merle Postel D’Aubigne. (MPD) 11 pacientes con excelentes resultados con puntaje obtenido en sus cinco rubros mayor de cinco puntos, uno de ellos malos resultados ya que no logra marcha y continua con dolor.

El seguimiento promedio fue de 38.3 meses (rango de uno a siete años), la escala de Merle Postel D’Aubigne. (MPD) 11 pacientes con excelentes resultados con puntaje obtenido en sus cinco rubros mayor de cinco puntos, uno de ellos tuvo malos resultados, ya que no logra la marcha y continua con dolor.

El seguimiento radiológico fue de dos, cuatro y ocho semanas y posteriormente bimensual con los siguientes re- sultados: 12 de las 13 fracturas tuvieron una consolidación y sólo un paciente presentó protrusión y aflojamiento del cotilo, pero no aceptó tratamiento quirúrgico, los 13 pacientes tuvieron una mínima migración vertical con una media de $2.6 \mathrm{~mm}$ y 2.1 migración horizontal a excepción de un paciente con más de $1.1 \mathrm{~cm}$ de migración.

Las complicaciones en este estudio fuero: un solo aflojamiento y dos luxaciones, las cuales se resolvieron con reducción bajo sedación, dos infecciones que remitieron y posteriormente se reintervinieron y se colocó anillo de reforzamiento.

\section{Discusión}

Las fracturas intraoperatorias acetabulares son una complicación extremadamente rara. De todas las revisiones de THA encontramos que $2-5 \%$ se deben a fracturas periprotésicas acetabulares. ${ }^{1,10}$ En nuestro estudio encontramos una tasa de incidencia de $.23 \%$, las 13 fracturas fueron en prótesis no cementadas, lo que se corrobora con la literatura que indica que se presentan mayormente en prótesis no cementadas. ${ }^{9}$ La elección de implante 
como punto importante demuestra que el uso de un cotilo hemisférico rígido aumenta $3.5 \%$ el riesgo y con el uso de hemisféricos disminuye a $0.009 \% .{ }^{11}$ En nuestro caso encontramos una alta incidencia con el uso de un cotilo CSF plus, que es 53\%. El cotilo CSF plus fabricado con aleación de titanio Ti-6Al-4V recubierta con cerámica de hidroxiapatita cuenta con un diseño esférico para fijarse a presión, ya que su diámetro ecuatorial es ligeramente superior al diámetro nominal, ${ }^{19}$ esto conlleva a que el cirujano al momento de impactarlo y querer llegar al fondo acetabular tenga una fractura acetabular.

\section{Conclusión}

Estas fracturas son raras, conocer el implante a utilizar es imperativo, ya que el reconocer que estén sobredimensionados, con mayor rigidez o hemisféricos tienen mayor riesgo de sufrir fracturas acetabulares. El tratamiento conservador con ocho semanas tiene un alto grado de consolidación y resultados funcionales excelentes, mientras que las fracturas inestables aumentan el riesgo de protrusiones acetabulares o de más cirugías de revisión.

Referencias

1. Clohisy JC, Calvert G, Tull F, McDonald D, Maloney WJ. Reasons for revision hip surgery: a retrospective review. Clin Orthop Relat Res. 2004; 429: 188-92. doi: 10.1097/01.blo.0000150126.73024.42.

2. Kurtz S, Ong K, Lau E, Mowat F, Halpern M. Projections of primary and revision hip and knee arthroplasty in the United States from 2005 to 2030. J Bone Joint Surg Am. 2007; 89(4): 780-5.

3. Bozic KJ, Kurtz SM, Lau E, et al. The epidemiology of revision total knee arthroplasty in the United States. Clin Orthop Relat Res. 2010; 468(1): 45-51.
4. Springer BD, Fehring TK, Griffin WL, Odum SM, Masonis JL. Why revision total hip arthroplasty fails. Clin Orthop Relat Res. 2009; 467(1): 166-73.

5. Curtis MJ, Jinnah RH, Wilson VD, Hungerford DS. The initial stability of uncemented acetabular components. J Bone Joint Surg Br. 1992; 74: 372-6.

6. Sharkey PF, Hozack WJ, Callaghan JJ, Kim YS, Berry DJ, Hanssen AD, LeWallen DG. Acetabular fracture associated with cementless acetabular component insertion: a report of 13 cases. J Arthroplasty. 1999; 14(4): 426-31.

7. Hansen TM, Koeneman JB, Hedley AK. 3-D FEM analysis of interference fixation of acetabular implants. Orthop Res Soc Trans. 1992; 17: 400.

8. Yerby SA, Taylor JK, Murzic WJ. Acetabular component interface: press-fit fixation. Trans Orthop Res Soc. 1992; 17: 384.

9. Della Valle CJ, Momberger NG, Paprosky WG. Periprosthetic fractures of the acetabulum associated with a total hip arthroplasty. Instr Course Lect. 2003; 52: 281-90.

10. Cook RE, Jenkins PJ, Walmsley PJ, et al. Risk factors for periprosthetic fractures of the hip: a survivorship analysis. Clin Orthop Relat Res. 2008; 466: 1652-6.

11. Haidukewych GJ, Jacofsky DJ, Hanssen AD, Lewallen DG. Intraoperative fractures of the acetabulum during primary total hip arthroplasty. J Bone Joint Surg Am. 2006; 88(9): 1952-6.

12. Desai G, Ries MD. Early postoperative acetabular discontinuity after total hip arthroplasty. J Arthroplasty. 2011; 26(8): 1570.e17-9.

13. McGrory BJ. Periprosthetic fracture of the acetabulumduring total hip arthroplasty in a patient with Paget's disease. Am J Orthop (Belle Mead NJ). 1999; 28(4): 248-50.

14. Callaghan JJ. Periprosthetic fractures of the acetabulum during and following total hip arthroplasty. Instr Course Lect. 1998; 47: 231-5.

15. Kim YS, Callaghan JJ, Ahn PB, Brown TD. Fracture of the acetabulum during insertion of an oversized hemispherical component. $J$ Bone Joint Surg Am. 1995; 77(1): 111-7.

16. Peterson CA, Lewallen DG. Periprosthetic fracture of the acetabulum after total hip arthroplasty. J Bone Joint Surg Am. 1996; 78(8): 1206-13.

17. Duncan CP, Masri BA. Fractures of the femur after hip replacement. Instr Course Lect. 1995; 44: 293-304.

18. Pascarella R, Sangiovanni P, Cerbasi S, et al. Periprosthetic acetabular fractures: a new classification proposal injury. Injury. 2018; 49S3: S65-S73

19. https://jri-ltd.com/wp-content/uploads/2017/10/019-SPANISH.pdf 